\title{
Multi-Objective Topology Optimization of Multi-Functional Components in Multibody Dynamics Systems
}

\author{
Guang Dong and Zheng-Dong Ma* \\ Mechanical Engineering Department, University of Michigan, Ann Arbor, MI, 48105, US
}

\begin{abstract}
In this paper, topology optimization technique is extended to consider multibody dynamics systems with a much more open design space, which can include passive, active, and reactive multi-functional components. General representative models for the multifunctional components are established in a multibody dynamics system. The topology optimization process has been advanced for the optimization of geometrically nonlinear, time-dependent, and timing-dependent multibody dynamics systems undergoing large nonlinear displacements with nonlinear dynamics responses as design objectives. The sensitivity analysis methods in this paper have made it possible to calculate the sensitivities in complicated multibody dynamic systems and provide users with choices to significantly reduce the computational costs, especially, in the topology optimization process, and to obtain desired accuracy in the sensitivity analysis. The single objective topology optimization problem can be redefined with multiple objectives, and solved using the same sensitivity analysis methods and the multi-objective optimization algorithm, such as global criterion method.
\end{abstract}

\section{Nomenclature}

$N \quad$ number of connecting members between multibody systems

$n$ number of rigid bodies in multibody systems

$f \quad$ interactive force between multibody systems

q Generalized coordinates vector

$k \quad$ Stiffness coefficient, $\mathrm{N} / \mathrm{m}$

$c \quad$ Damping coefficient, $\mathrm{N} /(\mathrm{m} / \mathrm{s})$

$m \quad$ Mass, $\mathrm{kg}$

$J \quad$ Moment of inertia, $\mathrm{kg} \cdot \mathrm{m}^{2}$

$\Delta \quad$ Relative distance change between interacting points in multibody systems, $\mathrm{m}$

$G \quad$ Design objective function

$h$ Design constraint function

$\alpha \quad$ Design variables

$\gamma \quad$ Grouping index

Subscript

$i \quad$ Number of order in the set

Superscript

$m \quad m$ th rigid body in multibody systems

$n \quad n$th rigid body in multibody systems

\footnotetext{
*Corresponding author: Research Scientist, ASME Fellow, mazd@umich.edu
} 


\section{Introduction}

\section{A. Background}

A structure with multi-functional components can be used in a wide range of applications. In this study, the word "multi-functional" will refer to a device with a combination of passive, active, and reactive components. For the purposes herein, a "passive" device is defined as a structure or device that responds to excitation passively without an active action. An "active" device is defined as a structure or device that can actively respond to the excitation, and which has an energy supply for the operation. A "reactive" structure is defined as a class of smart structures that can react to external excitations in a specifically designed way using energy that is either pre-stored in the system, or that comes from the external excitation, to counteract an undesirable situation or perform other desired tasks. ${ }^{1-4}$ A system that has multi-functional components may be made up of many multidisciplinary elements, including traditional mechanism components, hydraulic components, electromagnetic components, and so on. The designer needs to find an optimal layout based on these components in order to maximize/minimize single or multiple design objectives, in order to design the system to satisfy specific requirements.

Since the foundational work of Bendsøe and Kikuchi, ${ }^{5}$ the topology optimization method for optimal structural layout design has received extensive attention, as seen by its wide application to many structural optimization problems. ${ }^{6-11}$ In the ground structure approach developed by Zhou and Rozvany, ${ }^{12}$ a topology optimization problem is transformed to a problem of seeking the optimal layout in a design space that takes into consideration all the possible interactive members between the predefined nodal points and the optimization proceeds; this is done by removing unnecessary interactive members and reinforcing necessary interactive members in the design space in order to improve the design objective.

Since topology optimization is widely accepted as an efficient and successful layout optimization tool, it will be employed in this study. We initially assume that all the possible connections between the given multibody dynamics systems comprise the interactive system, and that it includes the open design space of passive, active, and reactive components. We then achieve the optimal layout by removing unnecessary multi-functional interactive members and reinforcing necessary multi-functional interactive members between the given multibody dynamics systems. Finally, the optimal multi-functional components system layout can be achieved as the interactive system between the given multibody dynamics systems. There exists a need to develop robust and efficient topology optimization approach that can be applied to a multi-functional components system. In this study, topology optimization technique is further extended to address the multibody dynamics system problems. In these systems, the components that are to be designed represent connections between large displacement, large rotation motions of the subsystems' bodies, and the connections can be achieved by using multi-functional components. Additionally, sensitivity analysis for topology optimization in a multibody dynamics system is more challenging than for a static or structural dynamic system.

\section{B. Motivation}

It is necessary to develop a methodology to obtain an optimally combined structural and material system, a system that makes the best use of passive, active, and reactive multi-functional members; and that optimizes the multiple design objectives in multibody dynamics systems.

This research highlights the following:

1. Dealing with design objectives that take into consideration the time-dependent, timing-dependent, large displacement dynamic nonlinear responses;

2. Establishing general representative models for the multi-functional (passive, active, and reactive) components in a multibody dynamics simulation system;

3. Designing a system that can optimally satisfy multiple requirements under widely varying operating conditions;

4. Developing efficient sensitivity analysis methods for the topology optimization of multibody dynamics systems;

5. Addressing the design problems with various system uncertainties. 


\section{Application}

This multi-functional components system layout optimization technology can be applied to various applications including vehicle transportation systems, ground and sea vehicle mooring systems, as well as space vehicle landing systems.

For a transportation system, the design objective can be the relative movement of the vehicle with respect to the carrier vehicle (ground, sea, or air) for the transportation operation in a dynamic environment in order to restrain the vehicle. The design space could include connecting chains, networked belts, or other constraint mechanisms.

For an optimal mooring system, the design objective could be the vessel's lateral and longitudinal accelerations, and its yawing movements in order to restrain the vessel. The design space can be seen as all the possible interactions between the vessel and the dock with the objective of finding the optimal mooring system.

For an occupant restraint system designs, such as the Gunner Restraint System(GRS), both the vehicle and the gunner can be subjected to significant relative and absolute motions under extreme driving or threatening external conditions. In addition, the restraint or connection components could employ amplitudedependent, time dependent, and timing-dependent behaviors, such as active belt retractors.

\section{Two General Multibody Dynamics Systems Connected by Multi-Functional Components}

As shown in Figure 1, it is assumed that two general Multibody Dynamics System(MDS), MDS-1 and MDS-2, are interconnected by a set of $N$ connecting members; this setup comprises the interactive system between these two given MDS. Each system has a number of rigid bodies linked by joints, bushings, and other internal constraints. It is assumed that there are $n_{1}$ rigid bodies in MDS- 1 , and $n_{2}$ rigid bodies in MDS-2. This set of connecting members represents a possible interactive system that restrains the relative motion between the two given MDS. Each member in the interactive system can be described as supplying a general interactive force between the interactive points, with one point on each of the two multibody dynamics systems. The interactive force may have non-linear dependency in relation to the relative kinematics (displacement, velocity, and acceleration) of the points, and it can be time-dependent or timing-dependent, or both. The components can be passive, active, or reactive, depending on the application. The layout of these connecting members will affect the system's dynamic responses, and the layout needs to be optimally designed for the specific objective function. This multibody dynamics systems are general enough for developing the methodology, and the number of multibody dynamics systems can be easily extended to more than two systems.

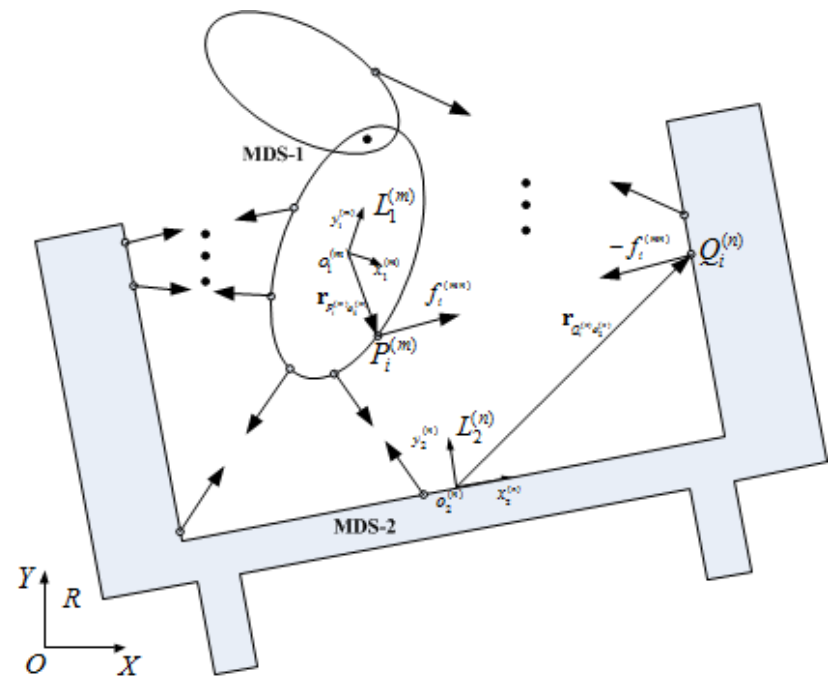

Figure 1. General multibody dynamic systems with interactive forces

In general, the $i$ th general interactive force $f_{i}$, which acts on $m$ th body in MDS- 1 , and on the $n$th body 
in MDS-2, can be described using

$$
f_{i}=f_{i}\left(\Delta_{i}, \dot{\Delta}_{i}, t, t_{i}^{0}, \delta_{i}^{0}, \boldsymbol{\kappa}_{i}\right)
$$

where the subscript $i$ denotes the $i$ th interactive member; $\Delta_{i}$ denotes the relative distance change (deformation) between the two interacting points as shown in Figure 1, in which $P_{i}^{(m)}$ is the connecting point of the $i$ th interactive member of the $m$ th body in the MDS- 1 ; and $Q_{i}^{(n)}$ is the connecting point of the $i$ th interactive member of the $n$th body in the MDS-2. $\dot{\Delta}_{i}$ denotes the speed (time directive of $\Delta_{i}$ ); $t_{i}^{0}$ denotes the critical timing for activating the $i$ th interactive member; $\delta_{i}^{0}$ denotes an initial distance gap for the $i$ th interactive member to become active; and $\boldsymbol{\kappa}_{i}$ is a vector of other design parameters for the $i$ th interactive member. For example, a simple form of $f_{i}$, which is a function only of deformation and velocity of deformation, is given by:

$$
f_{i}=k_{i} \Delta_{i}+c_{i} \dot{\Delta}_{i}
$$

where $k_{i}$ and $c_{i}$ are stiffness and damping coefficient for the $i$ th interactive member.

A one-way contact with an initial gap function $\delta_{i}^{0}$ can be described using:

$$
f_{i}=\left\{\begin{array}{cc}
0 & \left(\Delta_{i}<\delta_{i}^{0}\right) \\
k_{i}\left(\Delta_{i}-\delta_{i}^{0}\right)+c_{i} \dot{\Delta}_{i} & \left(\Delta_{i} \geq \delta_{i}^{0}\right)
\end{array}\right.
$$

An example of time dependent and timing dependent impulse interactive force function $f_{i}$ is

$$
f_{i}=f_{0 i} \exp \left(-\sigma_{0 i}\left(t-t_{0 i}\right)^{2}\right)
$$

where $f_{0 i}, \sigma_{0 i}$, and $t_{0 i}$ are design parameters for the $i$ th interactive member.

Since the $i$ th interactive member connects the $m$ th body in MDS- 1 to the $n$th body in MDS- $2, f_{i}$ can also be denoted as $f_{i}^{(m n)}$; and $\Delta_{i}$ can also be denoted as $\Delta_{i}^{(m n)}$. The direction of the interactive force $f_{i}^{(m n)}$ of the $i$ th member is defined by

$$
\mathbf{e}_{i}^{(m n)}=\frac{\mathbf{r}_{Q_{i}^{(n)} P_{i}^{(m)}}}{\| \mathbf{r}_{Q_{i}^{(n)} P_{i}^{(m)} \|}}
$$

where $\mathbf{r}_{Q_{i}^{(n)} P_{i}^{(m)}}$ denotes the vector of $\overline{P_{i}^{(m)} Q_{i}^{(n)}}$. Therefore, the $i$ th force vector acting on the MDS-1 is $\mathbf{f}_{i}^{1}=f_{i} \mathbf{e}_{i}$, and the force vector of the same interactive member acting on the MDS-2 is $\mathbf{f}_{i}^{2}=-f_{i} \mathbf{e}_{i}$. Then we have $\mathbf{f}_{i}^{1}+\mathbf{f}_{i}^{2}=\mathbf{0}$. The system's global force vector $\mathbf{F}_{g}$ and global deformation vector $\boldsymbol{\Delta}$ be given as:

$$
\begin{aligned}
& \mathbf{F}_{g}=\left[f_{1}, f_{2}, \ldots, f_{N}\right]^{T} \\
& \boldsymbol{\Delta}=\left[\Delta_{1}, \Delta_{2}, \ldots, \Delta_{N}\right]^{T}
\end{aligned}
$$

which represents the force and deformation vectors of the interactive system, with a total of $N$ interactive members.

Assume a global coordinate system $R: O-X Y Z$, and local coordinate systems $L_{1}^{(m)}: o_{1}^{(m)}-x_{1}^{(m)} y_{1}^{(m)} z_{1}^{(m)}$ with origin $o_{1}^{(m)}$ attached to the mass center of $m$ th body in MDS-1, $L_{2}^{(n)}: o_{2}^{(n)}-x_{2}^{(n)} y_{2}^{(n)} z_{2}^{(n)}$ with origin $o_{2}^{(n)}$ attached to the mass center of $n$th body in MDS-2. Assuming $\mathbf{q}_{1}=\left[\left(\mathbf{q}_{1}^{(1)}\right)^{T},\left(\mathbf{q}_{1}^{(2)}\right)^{T}, \ldots,\left(\mathbf{q}_{1}^{\left(n_{1}\right)}\right)^{T}\right]^{T}$ is the generalized coordinates vector of MDS-1, $\mathbf{q}_{2}=\left[\left(\mathbf{q}_{2}^{(1)}\right)^{T},\left(\mathbf{q}_{2}^{(2)}\right)^{T}, \ldots,\left(\mathbf{q}_{2}^{\left(n_{2}\right)}\right)^{T}\right]^{T}$ is the generalized coordinates vector of MDS-2, the governing equations for MDS-1 can be written as:

$$
\left\{\begin{array}{l}
\mathbf{M}_{1}\left(\mathbf{q}_{1}\right) \ddot{\mathbf{q}}_{1}-\mathbf{Q}_{v 1}+\left(\boldsymbol{\Phi}_{1}\right)_{\mathbf{q}_{1}}^{T} \boldsymbol{\lambda}_{1}=\mathbf{F}_{1}^{E x t}+\mathbf{F}_{1}^{q} \\
\boldsymbol{\Phi}_{1}\left(\mathbf{q}_{1}, t\right)=\mathbf{0}
\end{array}\right.
$$

where the first equation in Eq. 8 is the dynamic equilibrium equation, and the second equation is the constraint equation for MDS-1. $\mathbf{M}_{1}$ denotes the generalized mass matrix, $\left(\boldsymbol{\Phi}_{1}\right)_{\mathbf{q}_{1}}$ denotes the Jacobian matrix of $\boldsymbol{\Phi}_{1}, \boldsymbol{\lambda}_{1}$ denotes vector of Lagrangian multipliers. $\mathbf{Q}_{v 1}$ is the quadratic velocity term. $\mathbf{F}_{1}^{E x t}$ denotes the external force applied on MDS-1, $\mathbf{F}_{1}^{q}$ is the generalized force vector of MDS-1 due to the interactive system to be designed. 
Similarly, the governing equations for MDS-2 can be written as:

$$
\left\{\begin{array}{l}
\mathbf{M}_{2}\left(\mathbf{q}_{2}\right) \ddot{\mathbf{q}}_{2}-\mathbf{Q}_{v 2}+\left(\mathbf{\Phi}_{2}\right)_{\mathbf{q}_{2}}^{T} \boldsymbol{\lambda}_{2}=\mathbf{F}_{2}^{E x t}+\mathbf{F}_{2}^{q} \\
\boldsymbol{\Phi}_{2}\left(\mathbf{q}_{2}, t\right)=\mathbf{0}
\end{array}\right.
$$

where $\mathbf{M}_{2}$ denotes the generalized mass matrix; $\left(\boldsymbol{\Phi}_{2}\right)_{\mathbf{q}_{2}}$ denotes the Jacobian matrix of $\boldsymbol{\Phi}_{2} ; \boldsymbol{\lambda}_{2}$ denotes the vector of Lagrangian multipliers. $\mathbf{Q}_{v 2}$ is the quadratic velocity term. $\mathbf{F}_{2}^{E x t}$ denotes the external force applied on MDS-2, and $\mathbf{F}_{2}^{q}$ is the generalized force vector of MDS-2 that results from the designed interactive system.

$\mathbf{F}_{1}^{q}$ and $\mathbf{F}_{2}^{q}$ are the generalized force vectors defined in the generalized coordinate systems for MDS-1 and MDS-2. In general, $\mathbf{F}_{1}^{q}$ and $\mathbf{F}_{2}^{q}$ can be written as

$$
\left\{\begin{array}{l}
\mathbf{F}_{1}^{q}=\mathbf{B}_{1}^{T} \mathbf{F}_{g} \\
\mathbf{F}_{2}^{q}=\mathbf{B}_{2}^{T} \mathbf{F}_{g}
\end{array}\right.
$$

or equivalently,

$$
\left\{\begin{array}{l}
\mathbf{F}_{1}^{q} \\
\mathbf{F}_{2}^{q}
\end{array}\right\}=\mathbf{B}^{T} \mathbf{F}_{g}
$$

where $\mathbf{B}=\left[\begin{array}{ll}\mathbf{B}_{1} & \mathbf{B}_{2}\end{array}\right]$ is called Compatibility Matrix, which is a function of the generalized coordinates $\mathbf{q}_{1}$ and $\mathbf{q}_{2} \cdot \mathbf{B}_{1}$ is the compatibility matrix for MDS-1, and $\mathbf{B}_{2}$ is the compatibility matrix for MDS-2. Due to the effects of nonlinear geometry, the compatibility matrix $\mathbf{B}$ can be highly nonlinear with respect to $\mathbf{q}_{1}$ and $\mathbf{q}_{2}$. More details about the compatibility matrix $\mathbf{B}$ derivation can be found in Appendix.

\section{Topology Optimization for Multi-functional Components Design}

\section{A. Design variables}

An optimally combined structural or material system from a widely open design space that includes passive, active, and reactive components, will be found with respect to the design objectives based on the system's dynamic response $G(\mathbf{q}, \dot{\mathbf{q}}, \ddot{\mathbf{q}}, \boldsymbol{\alpha})$; this is done by solving a topology optimization problem efficiently. The optimization problem is defined based on state equations, general force elements, and critical boundary conditions. The design variables in this work are, $\boldsymbol{\alpha}=\left[\alpha_{1}, \alpha_{2}, \ldots, \alpha_{N}\right]^{T}, 0 \leq \alpha_{i} \leq 1(i=1,2, \ldots, N)$, which are similar to the relative density design variables in the power-law approach or Solid Isotropic Material with Penalization (SIMP) method; these are associated with each original global force element $f_{i}$. The design variables vector $\alpha$ also could be defined as cost functions or material coefficients. The modified global force element in the optimization problem $f_{i}^{*}$ is written as:

$$
f_{i}^{*}=\alpha_{i}^{\mu} f_{i}\left(0 \leq \alpha_{i} \leq 1, i=1,2, \ldots, N\right)
$$

where $\mu$ is the power parameter in topology optimization.

The global force vector $\mathbf{F}_{g}$ including the design variables $\boldsymbol{\alpha}$ for the interaction system can be rewritten in Eq. 13.

$$
\mathbf{F}_{g}=\left[\alpha_{1}^{\mu} f_{1}, \alpha_{2}^{\mu} f_{2}, \cdots, \alpha_{N}^{\mu} f_{N}\right]^{T}
$$

\section{B. Optimization problem definition}

In general, an objective function for multibody dynamics systems can be written as a function of generalized coordinates, generalized velocities, generalized accelerations, and design variables, namely, $G=G(\mathbf{q}, \dot{\mathbf{q}}, \ddot{\mathbf{q}}, \boldsymbol{\alpha})$. Topology optimization for the multibody dynamics systems with multi-function structural components with respect to dynamic response has the general form:

$$
\begin{gathered}
\min _{\boldsymbol{\alpha}} G(\mathbf{q}, \dot{\mathbf{q}}, \ddot{\mathbf{q}}, \boldsymbol{\alpha}) \\
\text { s.t. : state equations } \\
\sum_{i=1}^{N} \gamma_{i}^{j} \alpha_{i} V_{i} \leq h_{0 j} \quad(j=1,2, \ldots, m) \\
0 \leq \underline{\alpha}_{i} \leq \alpha_{i} \leq \bar{\alpha}_{i} \leq 1 \quad(i=1,2, \ldots, N) \\
\gamma_{i}^{j}: \text { grouping index } \quad\left(\gamma_{i}^{j}=0 \text { or } 1\right)
\end{gathered}
$$


where $m$ is the total number of constraint functions; $N$ is the total number of design variables; $V_{i}$ is the volume or cost function for the $i$ th design variable; and $h_{0 j}$ is the $j$ th constraint value. $\boldsymbol{\alpha}$ is the design variable vector, which is the material coefficient associated with each interactive member, similar to element relative densities in the SIMP method. The multi-functional components in the interaction system can be divided into different groups, each of which may belong to different disciplines, and each group can have its own constraint, resulting in a multi-domain design problem.

\section{Sensitivity analysis methods}

For practical topology optimization, an efficient sensitivity analysis method is critical because of the large number of design variables. Sensitivity analysis for topology optimization of multibody dynamics systems is quite different from topology optimization of a quasi-static system, since the governing equations of motion, and consequently the sensitivity analysis, are second-order differential equations. It is desirable to calculate a large number of sensitivities efficiently for every iteration based on the second-order differential-algebraic dynamics governing equations. For topology optimization of multibody dynamics systems, it is difficult to efficiently calculate a large number of sensitivities for every iteration, based on the second order differential governing equations with design variables. ${ }^{13,14}$

The state equations of a multibody dynamics system can be written in a general form: ${ }^{15}$

$$
\left\{\begin{array}{c}
\mathbf{r}=\mathbf{M}(\mathbf{q}) \ddot{\mathbf{q}}+\mathbf{F}_{t o t}(\mathbf{q}, \dot{\mathbf{q}}, t)+\boldsymbol{\Phi}_{\mathbf{q}}^{T} \boldsymbol{\lambda}=\mathbf{0} \\
\mathbf{\Phi}(\mathbf{q})=\mathbf{0}
\end{array}\right.
$$

where $\mathbf{r}$ is the residuals vector of dynamic equilibrium equation; $\mathbf{M}$ is the generalized mass matrix; $\mathbf{q}_{a \times 1}$ is generalized coordinates vector of multibody system; $\boldsymbol{\Phi}_{m \times 1}$ is the vector of linearly independent constraint equations; $\boldsymbol{\Phi}_{\mathbf{q}}$ is the constraint Jacobian matrix; $\boldsymbol{\lambda}$ is the vector of Lagrange multipliers; and $\mathbf{F}_{t o t}$ includes all the external forces term $\mathbf{Q}_{e}$ and quadratic velocity term $\mathbf{Q}_{v}$. In geometrically nonlinear dynamics system, $\mathbf{F}_{\text {tot }}$ could be highly nonlinear with respect to generalized coordinates $\mathbf{q}$.

Assuming a general form of the objective function $G$ for the optimization problem, then we have

$$
G=G(\ddot{\mathbf{q}}, \dot{\mathbf{q}}, \mathbf{q}, \boldsymbol{\lambda}, \boldsymbol{\alpha})=G(\mathbf{p}, \boldsymbol{\alpha})
$$

where,

$$
\mathbf{p}=\left[\ddot{\mathbf{q}}^{T}, \dot{\mathbf{q}}^{T}, \mathbf{q}^{T}, \boldsymbol{\lambda}^{T}\right]^{T}
$$

Differentiating the objective function in Eq. 16 with respect to the design variable vector $\boldsymbol{\alpha}$ obtains

$$
\frac{\mathrm{d} G}{\mathrm{~d} \boldsymbol{\alpha}}=\left[\frac{\partial G}{\partial \mathbf{p}}\right]^{T} \frac{\partial \mathbf{p}}{\partial \boldsymbol{\alpha}}+\frac{\partial f}{\partial \boldsymbol{\alpha}}
$$

Differentiating the multibody system equations in Eq. 15 with respect to the design variables $\boldsymbol{\alpha}$ obtains

$$
\left\{\begin{array}{l}
{\left[\frac{\partial \mathbf{r}}{\partial \mathbf{p}}\right]^{T} \frac{\partial \mathbf{p}}{\partial \boldsymbol{\alpha}}+\frac{\partial \mathbf{r}}{\partial \boldsymbol{\alpha}}=\mathbf{0}} \\
{\left[\frac{\partial \Phi}{\partial \mathbf{q}}\right]^{T} \frac{\partial \mathbf{q}}{\partial \boldsymbol{\alpha}}+\frac{\partial \mathbf{\Phi}}{\partial \boldsymbol{\alpha}}=\mathbf{0}}
\end{array}\right.
$$

which leads to the following equations

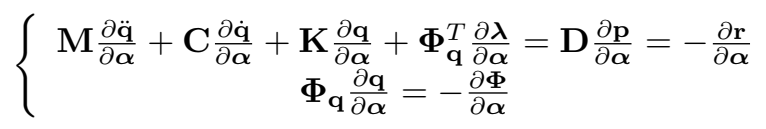

where

$$
\begin{gathered}
\mathbf{M}=\mathbf{M}(\mathbf{q}) ; \mathbf{C}=\frac{\partial \mathbf{r}}{\partial \dot{\mathbf{q}}}=\frac{\partial \mathbf{g}}{\partial \dot{\mathbf{q}}} ; \\
\mathbf{K}=\frac{\partial \mathbf{r}}{\partial \mathbf{q}}=\frac{\partial \mathbf{g}}{\partial \mathbf{q}}+\left[\frac{\partial \mathbf{M}}{\partial \mathbf{q}}\right] \ddot{\mathbf{q}}+\left[\frac{\partial \mathbf{\Phi}_{\mathbf{q}}^{T}}{\partial \mathbf{q}}\right] \boldsymbol{\lambda} \\
\boldsymbol{\Phi}_{\mathbf{q}}=\frac{\partial \mathbf{\Phi}}{\partial \mathbf{q}} ; \mathbf{D}=\left[\frac{\partial \mathbf{r}}{\partial \mathbf{p}}\right]^{T}=\left[\mathbf{M}, \mathbf{C}, \mathbf{K}, \boldsymbol{\Phi}_{\mathbf{q}}^{T}\right]
\end{gathered}
$$

The sensitivity analysis methods employed in this study for calculating $\frac{\mathrm{d} G}{\mathrm{~d} \boldsymbol{\alpha}}$ include the Adjoint Variable Method(AVM) method, the constant dynamic loading method, the time integration incorpated method based on the Generalized- $\alpha$ integration algorithm, and the iterative method. More details can be found in Dong and Ma's studies. ${ }^{14,16-18}$ 


\section{Optimization algorithm}

The General Sequential Approximate Optimization(GSAO) algorithm developed by Ma and Kikuchi ${ }^{10}$ is adopted to solve the topology optimization problem in Eq.14. This algorithm, based on convex approximation, extends the compatibility of previous optimization algorithms significantly by using advanced updating rules, and by offering more appropriate parameters for the optimization process algorithm. In specific cases, this algorithm reduces to other very popular topology algorithms. The GSAO enhancements result in improved convergence, higher computational efficiency, and a more stable iterative process for large-scale optimization problems. The flow chart of the GSAO optimization algorithm is shown in Figure 2.

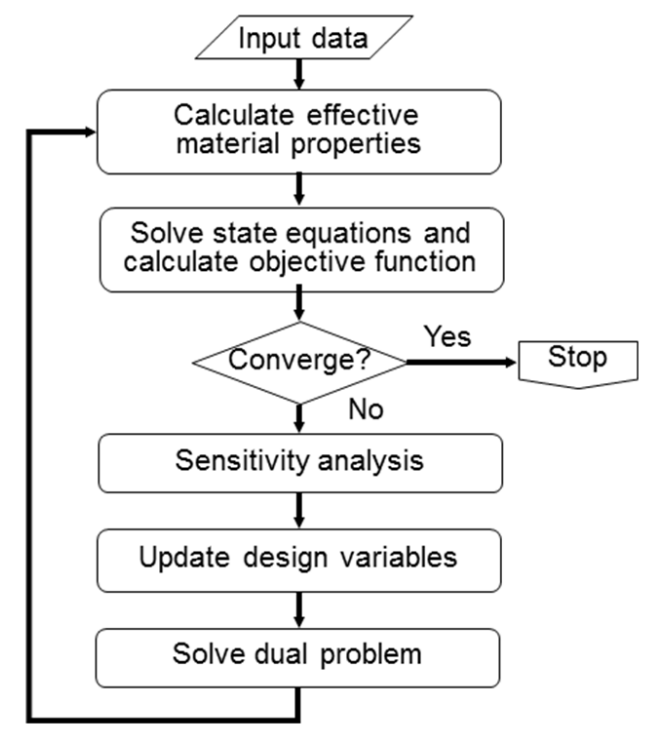

Figure 2. Flowchart of GSAO optimization algorithm ${ }^{10}$

\section{Numerical Examples}

\section{A. Two Multibody Dynamics Systems with Two Rigid Bodies}

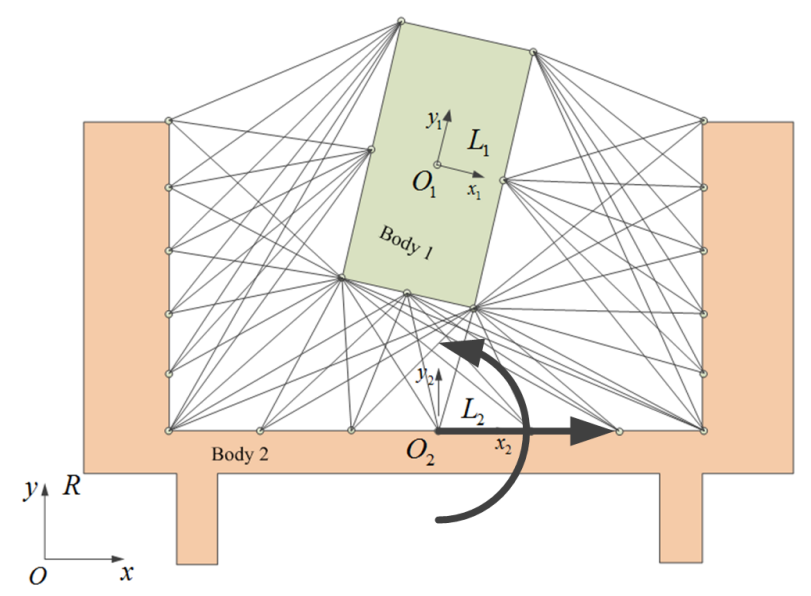

Figure 3. Two rigid bodies planar multibody dynamics model

A planar two multibody dynamics systems model is depicted, in which the two systems are connected by interactive members in Figure 3. The number of rigid bodies in each MDS is reduced to one. The Body 1 is in MDS-1, and the Body 2 is in MDS-2. The mass of Body 1 is $m_{1}=60 \mathrm{Kg}$ with the moment of 
inertia given as $J_{1}=10 \mathrm{Kg} \cdot \mathrm{m}^{2}$. The mass of Body 2 is $m_{2}=2000 \mathrm{Kg}$ with the moment of inertia given as $J_{2}=6000 \mathrm{Kg} \cdot \mathrm{m}^{2}$. There are 1020 interactive members between Body 1 and Body 2, with an initial linear stiffness of $22.5 \mathrm{~N} / \mathrm{m}$. Therefore, the interactive force elements have the form of Eq. 2 without the damping term. A rotational acceleration of magnitude $20 \mathrm{rad} / \mathrm{s}^{2}$ is applied to Body 2, with the rotation center of $\mathrm{O}_{2}$; a translational acceleration of magnitude $9.8 \mathrm{~m} / \mathrm{s}^{2}$ is applied to Body 2 along the global $x$ axis; and the system gravity acceleration is $9.8 \mathrm{~m} / \mathrm{s}^{2}$ with the negative global $y$ axis.

The design objective function is the maximum deviation energy stored in the whole interactive system during time duration $\left[t_{0}, t_{1}\right], G=\max _{\left[t_{0}, t_{1}\right]}\left\{g=\frac{1}{2} \boldsymbol{\Delta}^{T} \mathbf{K} \boldsymbol{\Delta}\right\}=\left.g(t)\right|_{t_{n}}$, which $g(t)$ is the sum of potential energy stored in all the interactive members of the interactive system with $t_{0}=0 \mathrm{~s}$ and $t_{1}=0.2 \mathrm{~s}, t_{n}$ is the global maximum point of the function $g(t)$. The design variables $\boldsymbol{\alpha}$ are normalized stiffness coefficient. The topology optimization problem for this multibody dynamics system is formulated as follows in Eq. 21:

$$
\begin{gathered}
\min _{\alpha_{i}(i=1,2, \ldots, N)}\left\{\max _{\left[t_{0}, t_{1}\right]} g=\frac{1}{2} \boldsymbol{\Delta}^{T} \mathbf{K} \boldsymbol{\Delta}\right\} \\
\text { s.t. : } \quad \text { state equations } \\
\sum_{i=1}^{N} \alpha_{i} V_{i} \leq h_{0} \\
0 \leq \underline{\alpha}_{i} \leq \alpha_{i} \leq \bar{\alpha}_{i} \leq 1 \quad(i=1,2, \ldots, N)
\end{gathered}
$$

The sensitivity analysis results of the AVM method, the time integration incorporated method and the iterative method for the initial design space $\left(\alpha_{i}=0.1\right)$ of first 51 interactive members are shown in Figure 4 , because the sensitivity pattern repeats for the rest of interactive members. Therefore, there are 51 points in the curves of sensitivities in Figure $4,\left.\left[\frac{\mathrm{d} G}{\mathrm{~d} \alpha_{1}} / \max \left\{\frac{\mathrm{d} G}{\mathrm{~d} \boldsymbol{\alpha}}\right\}, \frac{\mathrm{d} G}{\mathrm{~d} \alpha_{2}} / \max \left\{\frac{\mathrm{d} G}{\mathrm{~d} \boldsymbol{\alpha}}\right\}, \ldots, \frac{\mathrm{d} G}{\mathrm{~d} \alpha_{51}} / \max \left\{\frac{\mathrm{d} G}{\mathrm{~d} \boldsymbol{\alpha}}\right\}\right]\right|_{t=t_{n}}$. It is concluded that the iterative sensitivity analysis method gives the sensitivity same as the AVM method and the time integration incorporated method based on the Generalized- $\alpha$ algorithm. ${ }^{14,17,18}$

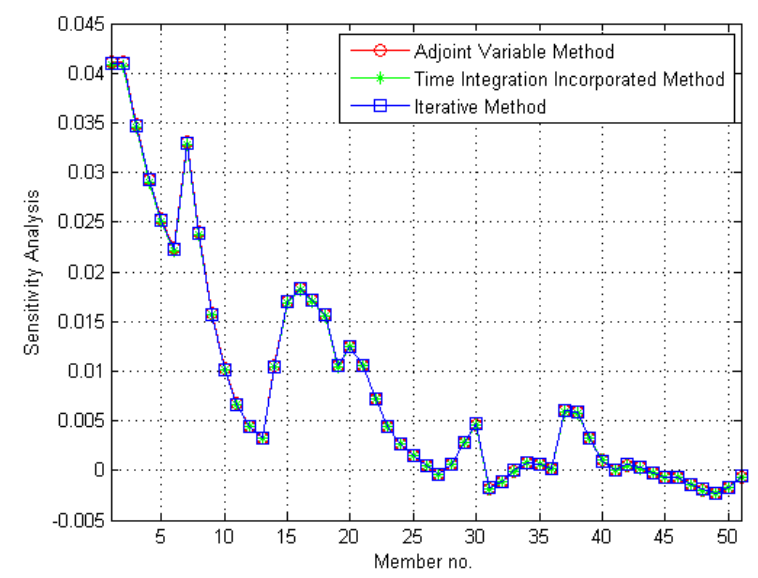

Figure 4. Sensitivity analysis results for the iterative method

Figure 5 shows the time history of sensitivities of first eight interactive members. It can be seen that the sensitivities calculated by the iterative method is same as the time integration incorporated method and the AVM method in all the time duration.

For each iteration using the iterative sensitivity analysis method, it uses 49.7 seconds, which is less than the AVM method 102.1 seconds, and the time integration incorporated method 54.8 seconds. The computer specification is in the Table $1 .^{14}$

The maximum iteration number is set at 50, and the optimization iteration results for the constant dynamic loading method, the AVM method, the time integration incorporated method and the iterative method are shown in Figure 6. From Figure 6, the proposed time integration incorporated sensitivity analysis method that uses the Generalized- $\alpha$ integration algorithm and the iterative sensitivity analysis method can, after 50 iterations, converge to the same optimization result as the AVM method. Nonetheless, the proposed 


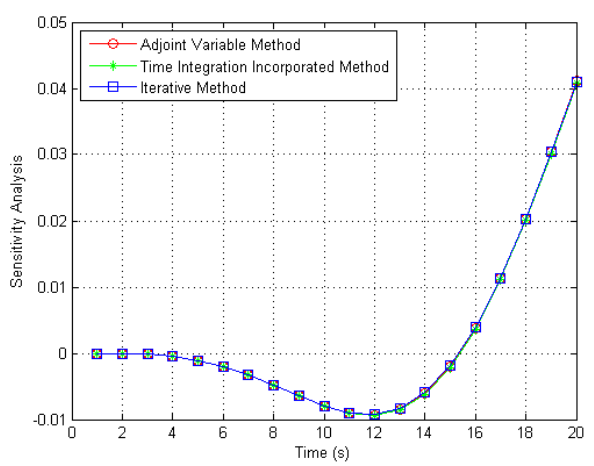

Interactive member 1

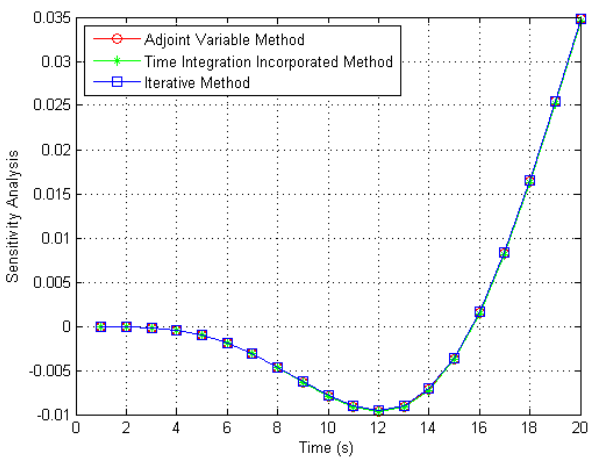

Interactive member 3

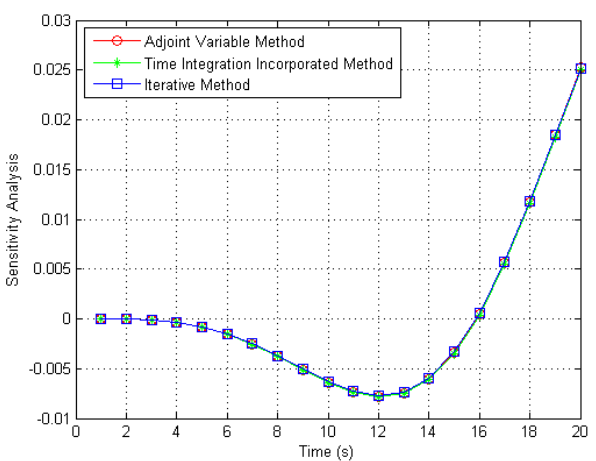

Interactive member 5

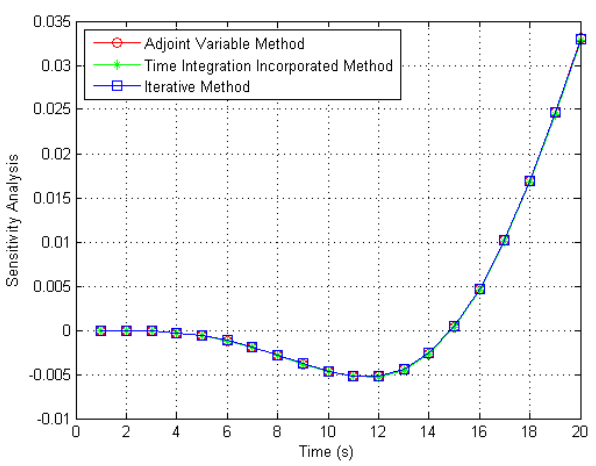

Interactive member 7

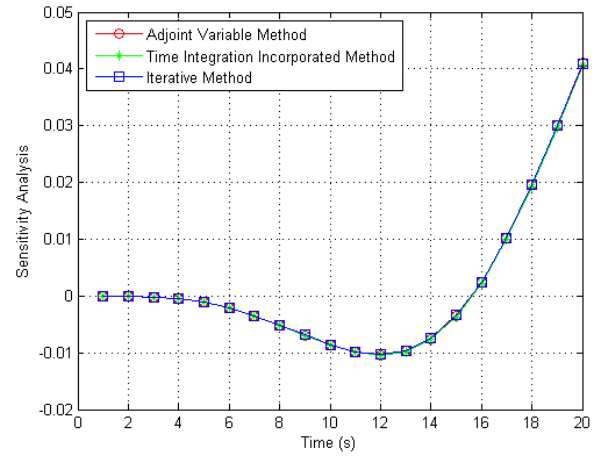

Interactive member 2

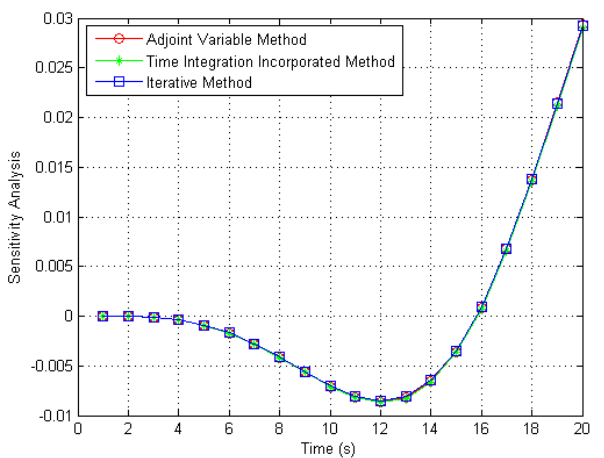

Interactive member 4

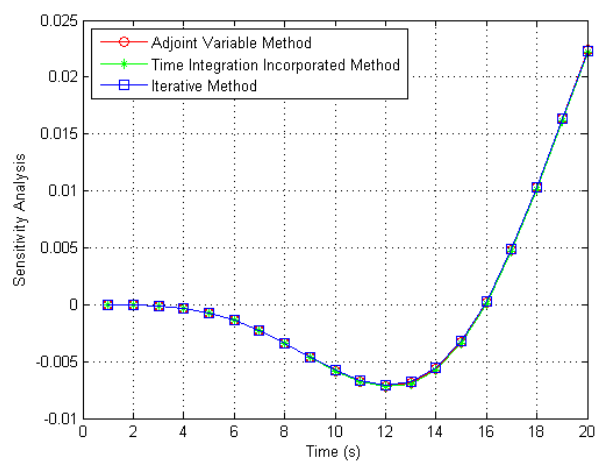

Interactive member 6

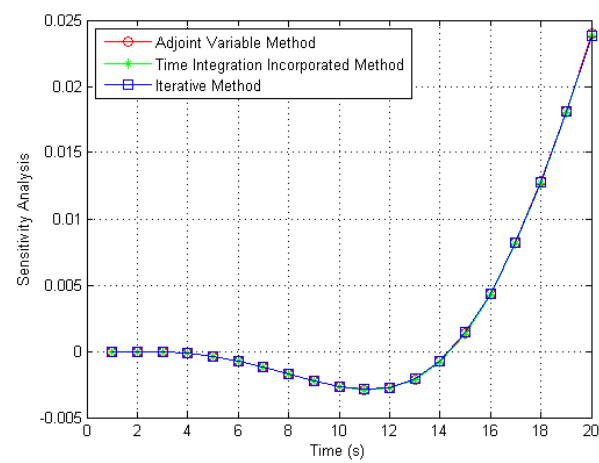

Interactive member 8

Figure 5. Sensitivity time history of first 8 members 
Table 1. Computer resource specification

\begin{tabular}{c|c} 
Processor & Intel Xeon $3.20 \mathrm{GHz}$ \\
\hline Memory & $8.00 \mathrm{~GB}$ \\
\hline Operating System & Windows 7 Enterprise version \\
\hline
\end{tabular}

constant dynamic loading sensitivity analysis can converge only to a result close to the AVM method with about 10\% difference after 50 iterations. These optimization results can also verify the conclusion in terms of accuracy of the proposed sensitivity analysis methods. The time integration incorporated and iterative sensitivity analysis methods are thus seen to be able to obtain the same sensitivities as the AVM method and converge to the same result, but much more efficient.

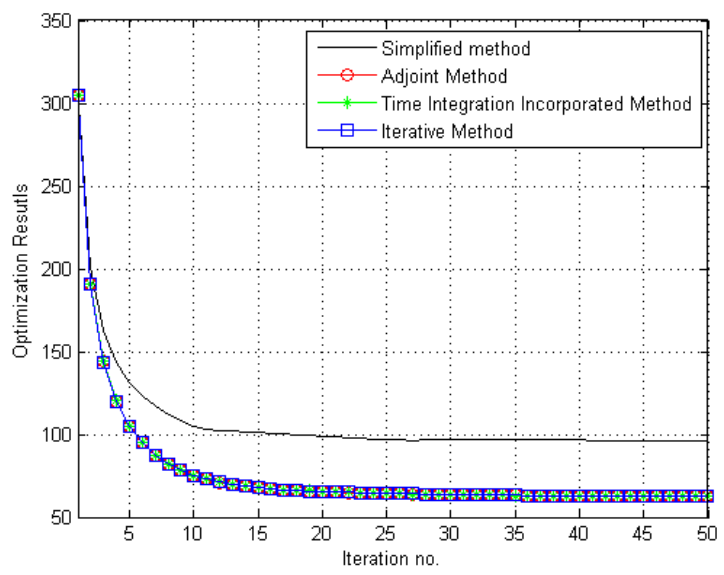

Figure 6. Optimization results for two bodies multibody dynamics model

\section{B. Two Multibody Dynamics Systems Multi-Objective Optimization}

The Multi-Objective Optimization(MOO) problem can be defined as follows in general:

$$
\begin{gathered}
\min _{\alpha_{i}(i=1,2, \ldots, N)} \mathbf{G}=\left[G_{1}(\boldsymbol{\alpha}), G_{2}(\boldsymbol{\alpha}), \ldots, G_{k}(\boldsymbol{\alpha})\right]^{T} \\
\text { s.t. }: h_{j}(\boldsymbol{\alpha}) \leq 0(j=1,2, \ldots, m)
\end{gathered}
$$

where $N$ is the number of design variables; $k$ is the number of objective functions; and $m$ is the number of constraint functions. Since the vector of design variables $\boldsymbol{\alpha} \in \mathbb{R}^{N}$, the vector of objective functions $\mathbf{G} \in \mathbb{R}^{k}$, and the single objective function $G_{i}(\boldsymbol{\alpha}): \mathbb{R}^{N} \rightarrow \mathbb{R}^{1}$. The feasible design space $\boldsymbol{\Gamma}$, which is also called the feasible decision space or constraint set, is defined as $\boldsymbol{\Gamma}=\left\{\boldsymbol{\alpha} \mid h_{j}(\boldsymbol{\alpha}) \leq 0 \quad j=1,2, \ldots, m\right\}$. The feasible criterion space $\boldsymbol{\Xi}$, which is also called the feasible cost space or the attainable set, is defined as $\boldsymbol{\Xi}=\{\mathbf{G}(\boldsymbol{\alpha}) \mid \boldsymbol{\alpha} \in \boldsymbol{\Gamma}\} .{ }^{19}$

Compared to the single-objective optimization, there is typically no single global solution for a multiobjective problem typically. It is often necessary to determine a set of points that all fit a predetermined definition for an optimum. The predominant concept in defining an optimal point is that of Pareto optimality. ${ }^{20}$

There are many sophisticated developed methods that employ a priori articulation of preferences, including the weighted global criterion method, the weighted sum method, the weighted min-max method, and the weighted product method, that also employ a posteriori articulation of preference, such as Normal Boundary Intersection(NBI) method, ${ }^{21}$ Normal Constraint(NC) method ${ }^{22}$ and Genetic Algorithm(GA) method. ${ }^{23}$ One general methods for MOO is the global criterion method in which all objective functions are combined to form a single function; all the optimization techniques for the usual single-objective function can then 
be applied. One of the most general utility functions using the global criterion method is expressed in its simplest form as the following weighted exponential summation: ${ }^{19}$

$$
U=\sum_{i=1}^{k} w_{i}\left[G_{i}(\boldsymbol{\alpha})\right]^{p}, \quad G_{i}(\boldsymbol{\alpha})>0 \forall i
$$

where $\mathbf{w}=\left[w_{1}, \ldots, w_{i}, \ldots, w_{k}\right]$ is the weights vector usually selected by the decision-makers such that $\sum_{i=1}^{k} w_{i}=1$ and $\mathbf{w}>\mathbf{0}$. Generally, the relative value of the weights reflects the relative importance of the design objectives.

The transformations of the original objective functions $G_{i}(\boldsymbol{\alpha})$ are widely used in the Eq. 23 because of the many advantages offered thereby. ${ }^{24}$ This is especially true with scalarization methods that involve a prior articulation of preferences. ${ }^{19}$ Moreover, the most robust approach to transforming objective functions, regardless of their original range, is given in Eq. $24:{ }^{25,26}$

$$
G_{i}^{\text {trans }}=\frac{G_{i}(\boldsymbol{\alpha})-G_{i}^{\circ}}{G_{i}^{\text {max }}-G_{i}^{\circ}}
$$

where $G_{i}^{\max }$ is the maximum of the design objective $G_{i}$ in the feasible design space $\boldsymbol{\Gamma}$, and $G_{i}^{\circ}$ is the utopia point of the design objective $G_{i}$. This approach is consistently referred to as normalization. In this case, $G_{i}^{\text {trans }}$ generally has values between zero and one, depending on the accuracy and method with which $G_{i}^{\max }$ and $G_{i}^{\circ}$ are determined.

Consider again the two multibody dynamics systems model with one rigid body in each system, in which the systems are connected by interactive members, as in the interactive system in Figure 3. All the system parameters were given in section A. There are 1020 interactive members between Body 1 and Body 2, with an initial linear stiffness of $22.5 \mathrm{~N} / \mathrm{m}$. The design objective function is the maximum deviation energy stored in the whole system in time duration of $\left[t_{0}, t_{1}\right]$. Two different loading conditions, however, are applied to the model; the first loading condition is a rotational acceleration that is applied to Body 2 with a magnitude of $20 \mathrm{rad} / \mathrm{s}^{2}$, with the rotation center of $\mathrm{O}_{2}$ without translation acceleration; the system gravity acceleration is $9.8 \mathrm{~m} / \mathrm{s}^{2}$ along negative global $y$ axis. The second loading condition is a translational acceleration that is applied to Body 2 with a magnitude of $20 \mathrm{~m} / \mathrm{s}^{2}$ along global $x$ axis without rotational acceleration. Because the objective function needs to be minimized under two different loading conditions, this problem can be treated as a MOO problem and formulated as follows in Eq. 25:

$$
\begin{gathered}
\min _{\alpha_{i}(i=1,2, \ldots, N)}\left\{G_{1}, G_{2}\right\} \\
\text { s.t. : } \quad \text { state equations } \\
\sum_{i=1}^{N} \alpha_{i} V_{i} \leq h_{0} \\
0 \leq \underline{\alpha}_{i} \leq \alpha_{i} \leq \bar{\alpha}_{i} \leq 1 \quad(i=1,2, \ldots, N)
\end{gathered}
$$

where $G_{1}=\max _{\left[t_{0}, t_{1}\right]}\left\{g=\frac{1}{2} \boldsymbol{\Delta}^{T} \mathbf{K} \boldsymbol{\Delta}\right\}$ under the first loading condition of pure rotation,

and $G_{2}=\max _{\left[t_{0}, t_{1}\right]}\left\{g=\frac{1}{2} \boldsymbol{\Delta}^{T} \mathbf{K} \boldsymbol{\Delta}\right\}$ under the second loading condition of pure translation.

The maximum iteration number is set at 100. The optimization iteration results in the criterion space for the time integration incorporated sensitivity analysis method and the iterative sensitivity analysis method are shown in Figure 7.

From Figure 7, the pareto frontier is clearly shown in the design space, then we can conclude that both time integration and iterative sensitivity analysis methods can solve this multi-objective topology optimization problem efficiently with the weighted exponential summation global criterion method. The final optimized design variables after 100 iterations for the Pareto point in the black circle in Figure 7 are shown in Figure 8.

\section{Conclusion}

A fundamental multi-functional components layout design technology based on topology optimization is proposed for a multibody dynamics systems design problem. It may have various options associated 


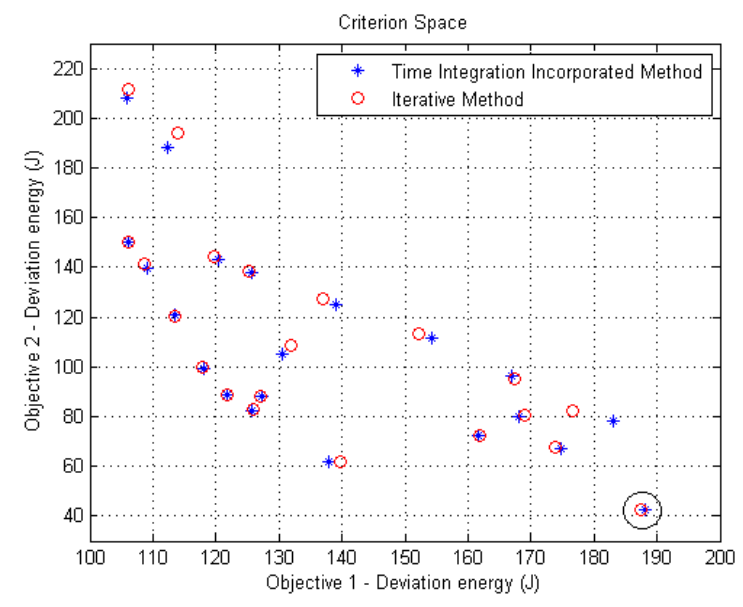

Figure 7. Optimization results for two bodies multibody dynamics model with two objectives

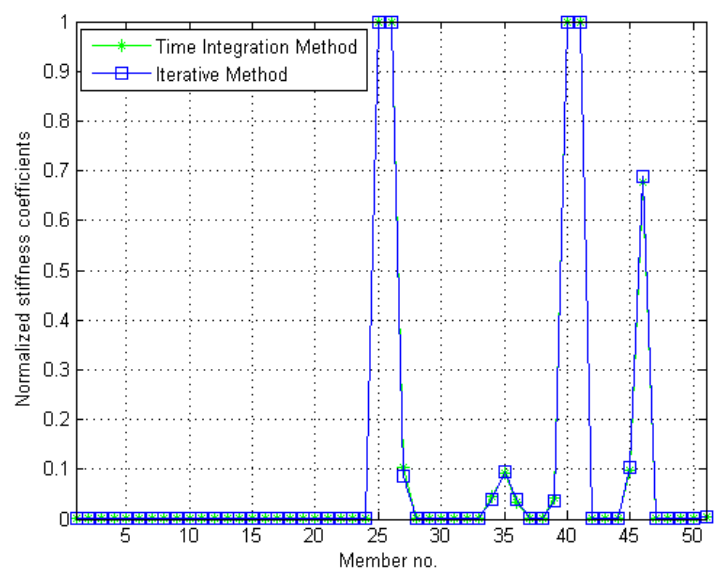

Figure 8. Optimized design variables for two bodies multibody dynamics model with two objectives 
with using passive, active, and reactive devices or components. The proposed topology optimization design method can address the objective functions that are related to dynamic responses of multibody dynamics systems rather than static responses, and that satisfy multiple requirements; the requirements may include designing a vehicle occupant restraint system that must perform under various operating conditions, and must meet stringent performance requirements. The proposed advanced topology optimization technique can also solve the multi-objective problems using the efficient sensitivity analysis techniques developed previously combined with appropriate MOO algorithms.

\section{Appendix}

In a planar multibody dynamics system as shown in Figure 1 , for the $m$ th body with generalized coordinates $\mathbf{q}_{1}^{(m)}=\left[x_{o_{1}^{(m)}}, y_{o_{1}^{(m)}}, \psi_{1}^{(m)}\right]^{T}$ in MDS-1, and the $n$th body with generalized coordinates $\mathbf{q}_{2}^{(n)}=$ $\left[x_{o_{2}^{(n)}}, y_{o_{2}^{(n)}}, \psi_{2}^{(n)}\right]^{T}$ in MDS-2. Then the first equation of Eq. 8 and 9 for the $m$ th body in MDS- 1 and the $n$th body in MDS-2 can be written in the following Newton-Euler form: ${ }^{27}$

$$
\begin{aligned}
& {\left[\begin{array}{ccc}
M_{1}^{(m)} & 0 & 0 \\
0 & M_{1}^{(m)} & 0 \\
0 & 0 & J_{1}^{(m)}
\end{array}\right]\left[\begin{array}{c}
\ddot{x}_{o_{1}^{(m)}} \\
\ddot{y}_{o_{1}^{(m)}} \\
\ddot{\psi}_{1}^{(m)}
\end{array}\right]=} \\
& {\left[\begin{array}{c}
\sum_{i_{m} \in I_{1}^{(m)}}\left(F_{i_{m}}^{q_{1}}\right)_{x} \\
\sum_{i_{i_{m} \in I_{1}^{(m)}}^{(m)}}\left(F_{i_{m}}^{q_{1}}\right)_{y} \\
\sum_{i_{1}^{(m)}}\left[-y_{P_{i_{m}}^{(m)} o_{1}^{(m)}}^{L_{(m)}^{(m)}} x_{\left.P_{i_{m}}^{(m)} o_{1}^{(m)}\right]}^{L^{(m)}}\right] \mathbf{A}^{L_{1}^{(m)} R_{[}}\left[\left(F_{i_{m}}^{q_{1}}\right)_{x}\left(F_{i_{m}}^{q_{1}}\right)_{y}\right]^{T}
\end{array}\right]+\left[\begin{array}{l}
\left(F_{E x t}^{(m)}\right)_{x} \\
\left(F_{E x t}^{(m)}\right)_{y} \\
M n t_{E x t}^{(m)}
\end{array}\right]} \\
& {\left[\begin{array}{ccc}
M_{2}^{(n)} & 0 & 0 \\
0 & M_{2}^{(n)} & 0 \\
0 & 0 & J_{2}^{(n)}
\end{array}\right]\left[\begin{array}{c}
\ddot{x}_{o_{2}^{(n)}} \\
\ddot{y}_{o_{2}^{(n)}} \\
\ddot{\psi}_{2}^{(n)}
\end{array}\right]=} \\
& {\left[\begin{array}{c}
\sum_{i_{n} \in I_{2}^{(n)}}\left(F_{i_{n}}^{q_{2}}\right)_{x} \\
\sum_{\sum_{n} \in I_{2}^{(n)}}\left[-y_{Q_{i_{n}}^{(n)} o_{2}^{(n)}}^{L_{(n)}^{(n)}}\left(F_{i_{n}}^{q_{2}}\right)_{y}\right. \\
\left.x_{i_{n} \in I_{2}^{(n)}}^{L_{2}^{(n)} o_{2}^{(n)}}\right] \mathbf{A}^{L_{2}^{(n)} R}\left[\left(F_{i_{n}}^{q_{2}}\right)_{x}\left(F_{i_{n}}^{q_{2}}\right)_{y}\right]^{T}
\end{array}\right]+\left[\begin{array}{l}
\left(F_{E x t}^{(n)}\right)_{x} \\
\left(F_{E x t}^{(n)}\right)_{y} \\
M n t_{E x t}^{(n)}
\end{array}\right]}
\end{aligned}
$$

where $M_{1}^{(m)}, M_{2}^{(n)}$ are the mass of the $m$ th body in MDS- 1 and the $n$th body in MDS-2. $J_{1}^{(m)}$ and $J_{2}^{(n)}$ are the moment of inertia with respect to mass center of the $m$ th body and the $n$th body respectively. Assuming there are $N_{m}$ interactive forces applied on the $m$ th body in MDS- 1 , the indexes of these forces elements are denoted as $I_{1}^{(m)}=\left\{i_{1}^{(m)}, i_{2}^{(m)}, \ldots, i_{N_{m}}^{(m)}\right\}$, similarly, for the $n$th body in MDS-2 we can define $I_{2}^{(n)}=\left\{i_{1}^{(n)}, i_{2}^{(n)}, \ldots, i_{N_{n}}^{(n)}\right\}$. Assuming that the interactive forces apply between the $m$ th body in MDS-1 and the $n_{1}$ th body, $n_{2}$ th body, $\ldots, n_{N_{m}}$ th body in MDS-2, then the global force vector for the $m$ th body in MDS-1 can be written as $\mathbf{F}_{g 1}^{(m)}=\left[f_{i_{1}^{(m)}}^{\left(m n_{1}\right)}, f_{i_{2}^{(m)}}^{\left(m n_{2}\right)}, \ldots, f_{i_{N_{m}}^{(m)}}^{\left(m n_{N_{m}}\right)}\right]^{T}$ in which $\mathbf{F}_{i_{m}}^{q_{1}}$ and $\mathbf{F}_{i_{n}}^{q_{2}}$ are generalized forces of the $i_{m}$ th interactive member for the $m$ th body in MDS- 1 and the $i_{n}$ th interactive member for the $n$th body in MDS-2, expressed in the global coordinate system. Note that

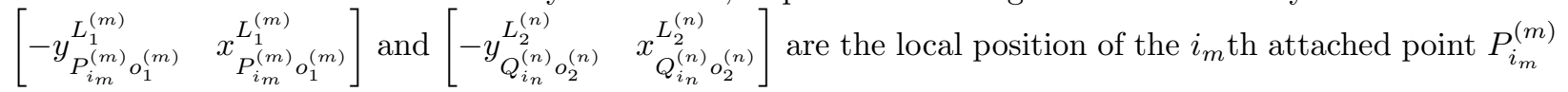


on the $m$ th body in MDS- 1 and the local position of the $i_{n}$ th attached point $Q_{i_{n}}^{(n)}$ on the $n$th body in MDS-2. $\left[\left(F_{E x t}^{(m)}\right)_{x},\left(F_{E x t}^{(m)}\right)_{y}, M n t_{E x t}^{(m)}\right]^{T}$ and $\left[\left(F_{E x t}^{(n)}\right)_{x},\left(F_{E x t}^{(n)}\right)_{y}, M n t_{E x t}^{(n)}\right]^{T}$ are the external force vectors applied on the respective $m$ th body in MDS- 1 and $n$th body in MDS- $2 . \mathbf{A}^{L_{1}^{(m)} R}$ and $\mathbf{A}_{2}^{L_{2}^{(n)} R}$ are the transformation matrix between local coordinate system $L_{1}^{(m)}, L_{2}^{(n)}$ and global coordinates system $R$. For the planar multibody dynamics systems, we have

$$
\begin{aligned}
\mathbf{A}^{L_{1}^{(m)} R} & =\left[\begin{array}{cc}
\cos \psi_{1}^{(m)} & \sin \psi_{1}^{(m)} \\
-\sin \psi_{1}^{(m)} & \cos \psi_{1}^{(m)}
\end{array}\right] \\
\mathbf{A}^{L_{2}^{(n)} R} & =\left[\begin{array}{cc}
\cos \psi_{2}^{(n)} & \sin \psi_{2}^{(n)} \\
-\sin \psi_{2}^{(n)} & \cos \psi_{2}^{(n)}
\end{array}\right]
\end{aligned}
$$

The $i$ th interactive force, which connects the $m$ th body in MDS- 1 and the $n$th body in MDS- 2 , can be expressed in the global system $R$ as follows,

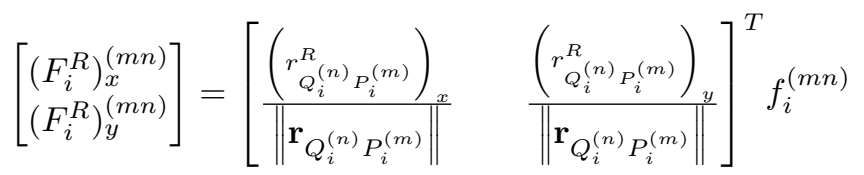

Therefore, the global force vector applied to the $m$ th body in MDS-1 can be denoted as $\mathbf{F}_{g 1}^{(m)}=$ $\left[f_{i_{1}^{(m)}}^{\left(m n_{1}\right)}, f_{i_{2}^{(m)}}^{\left(m n_{2}\right)}, \ldots, f_{i_{N_{m}}^{(m)}}^{\left(m n_{N_{m}}\right)}\right]^{T}$ and calculated as:

$$
\begin{aligned}
& {\left[\begin{array}{c}
\sum_{i_{n} \in I_{2}^{(n)}}\left(F_{i_{n}}^{q_{2}}\right)_{x} \\
\sum_{i_{n} \in I_{2}^{(n)}}^{\left(F_{n}\right.}\left(F_{i_{n}}^{q_{2}}\right)_{y} \\
\sum_{i_{n} \in I_{2}^{(n)}}\left[-y_{Q_{i_{n}}^{(n)} o_{2}^{(n)}}^{L_{(n)}^{(n)}} x_{Q_{i_{n}}^{(n)} o_{2}^{(n)}}^{L^{(n)}}\right] \mathbf{A}^{L_{2}^{(n)} R}\left[\left(F_{i_{n}}^{q_{2}}\right)_{x}\left(F_{i_{n}}^{q_{2}}\right)_{y}\right]^{T}
\end{array}\right]=\left(\mathbf{B}_{1}^{(m)}\right)^{T}\left[\begin{array}{c}
f_{i_{1}^{(m)}}^{\left(m n_{1}\right)} \\
f_{i_{2}^{(m)}}^{\left(m n_{2}\right)} \\
\vdots \\
f_{i_{N m}^{(m)}}^{\left(m n_{N_{m}}\right)}
\end{array}\right]} \\
& =\left(\mathbf{B}_{1}^{(m)}\right)^{T} \mathbf{F}_{g 1}^{(m)}
\end{aligned}
$$

where the compatibility matrix

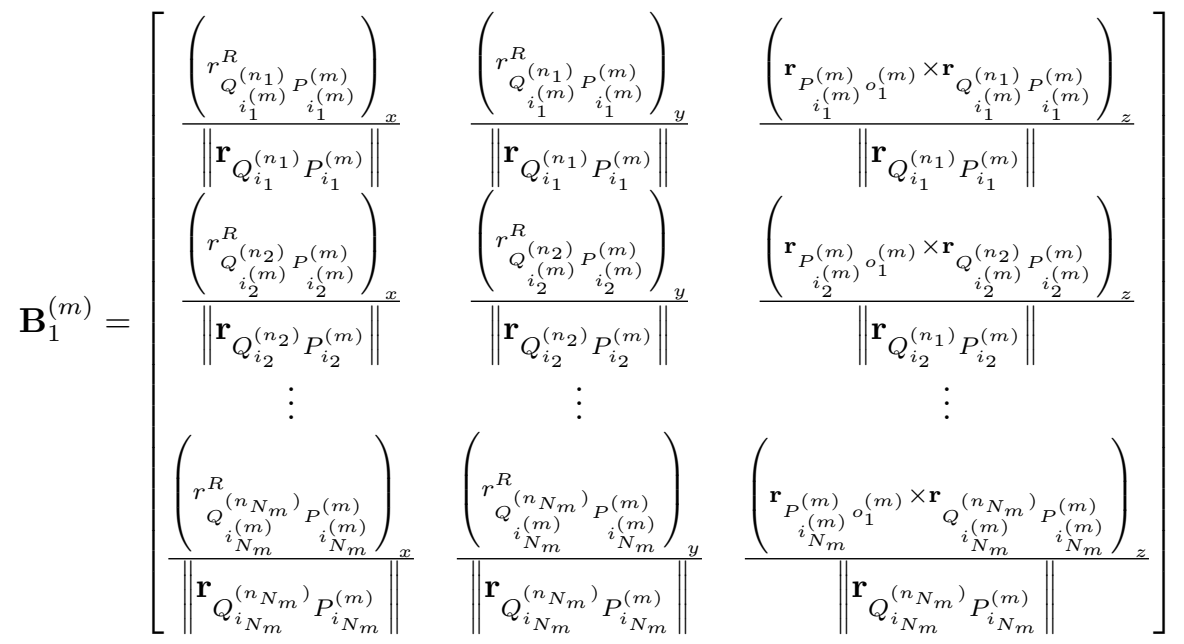

The relationship between the $m$ th compatibility matrix $\mathbf{B}_{1}^{(m)}$ in MDS-1, and the generalized coordinates 
$\mathbf{q}_{1}^{(m)}$ and $\mathbf{q}_{2}^{(n)}$ are, in general, highly nonlinear.

$$
\begin{aligned}
& \mathbf{r}_{Q_{i}^{(n)} P_{i}^{(m)}}=\left[\begin{array}{l}
\left(r_{Q_{i}^{R}}^{R(n)} P_{i}^{(m)}\right)_{x} \\
\left(r_{Q_{i}^{(n)} P_{i}^{(m)}}^{R}\right)_{y}
\end{array}\right]=
\end{aligned}
$$

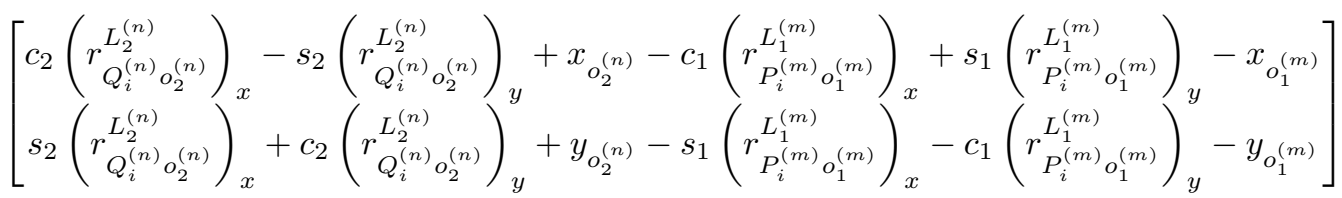

where $c_{2}=\cos \psi_{2}^{(n)} ; s_{2}=\sin \psi_{2}^{(n)} ; c_{1}=\cos \psi_{1}^{(m)} ;$ and $s_{1}=\sin \psi_{1}^{(m)}$.

Substituting Eq. 33 into 32, we obtain the nonlinear dependence of the compatibility matrix on the generalized coordinates.

Due to the large translation, large rotation, and nonlinear geometric properties of dynamics systems, the relationship between the deformation of the $i$ th connecting member $\Delta_{i}^{(m n)}$ and the generalized coordinates $\mathbf{q}_{1}^{(m)}=\left[x_{o_{1}^{(m)}}, y_{o_{1}^{(m)}}, \psi_{1}^{(m)}\right]^{T}$ for the $m$ th body and the $\mathbf{q}_{2}^{(n)}=\left[x_{o_{2}^{(n)}}, y_{o_{2}^{(n)}}, \psi_{2}^{(n)}\right]^{T}$ for the $n$th body can be highly nonlinear. The deformation of the $i$ th interactive member attached to the $m$ th body in MDS- 1 and the $n$th body in MDS- 2 is:

$$
\begin{aligned}
& \Delta_{i}^{(m n)}=\left\|\mathbf{r}_{Q_{i}^{(n)} P_{i}^{(m)}}\right\|-\left\|\mathbf{r}_{Q_{i}^{(n)} P_{i}^{(m)}}\right\|_{t=t_{0}} \\
& =\left\|\mathbf{A}^{R L_{1}^{(m)}} \mathbf{r}_{P_{i}^{(m)} o_{1}^{(m)}}^{L_{1}^{(m)}}+\mathbf{r}_{o_{1}^{(m)}}^{R}-\mathbf{A}^{R L_{2}^{(n)}} \mathbf{r}_{Q_{i}^{(n)} o_{2}^{(n)}}^{L_{2}^{(n)}}-\mathbf{r}_{o_{2}^{(n)}}^{R}\right\|-l_{i}^{0}
\end{aligned}
$$

Then, the deformation vector $\boldsymbol{\Delta}^{(m)}$ for the $m$ th body is denoted as

$$
\boldsymbol{\Delta}^{(m)}=\left[\Delta_{i_{1}^{(m)}}^{\left(m n_{1}\right)}, \Delta_{i_{2}^{(m)}}^{\left(m n_{2}\right)}, \cdots, \Delta_{i_{N_{m}}^{(m)}}^{\left(m n_{N_{m}}\right)}\right]^{T}
$$

The following relationship between the $m$ th deformation vector $\boldsymbol{\Delta}^{(m)}$ and the $m$ th compatibility matrix $\mathbf{B}_{1}^{(m)}$ is obtained by differentiating Eq. 35 with respect to the generalized coordinates $\mathbf{q}_{1}^{m}$ for the $m$ th body:

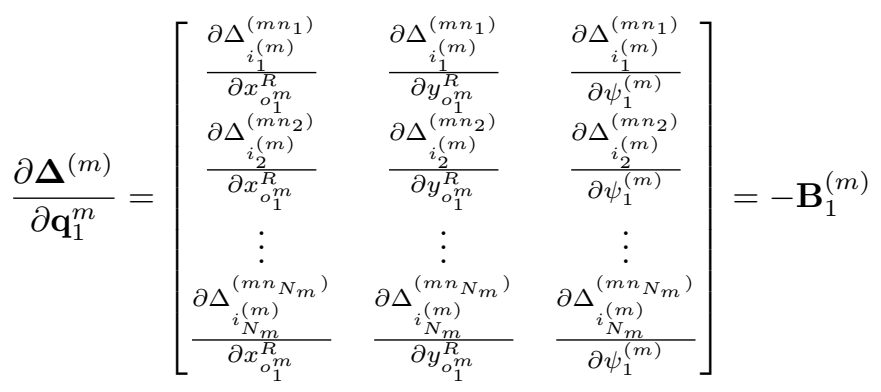

\section{Acknowledgments}

This research was supported partially by the Automotive Research Center, a US Army Center of Excellence headquartered at the University of Michigan. This support is gratefully acknowledged.

\section{References}

\footnotetext{
${ }^{1}$ Chiyo, D., Kodandaramaiah, S. B., Grosh, K., Ma, Z. D., Raju, B., and Abadi, F. R., "Reactive Structure and Smart Armor for Army's Future Ground Vehicles," Proceedings of the 27th Army Science Conference, Orlando, FL, Nov. 2010.

${ }^{2}$ Dong, G., Ma, Z. D., Hulbert, G. M., and Kikuchi, N., "Function-Oriented Material Design for an Innovative Gunner Restraint System," Proceedings of the 15th Automotive Research Center Annual Conference, Ann Arbor, MI, May 2009.

${ }^{3}$ Ma, Z.-D., Dong, G., Hope, K., and Arepally, S., "Function-Oriented Material Design for an Innovative Gunner Restraint System," Proceedings of Modeling and Simulation, Testing and Validation (MSTV) Conference 2008, Warren, MI, Nov. 2008.
} 
${ }^{4}$ Ma, Z.-D., Dong, G., Hulbert, G. M., Kikuchi, N., Arepally, S., Hope, K., Vunnam, M., Sheng, J., Luo, K., and Wang, H., "Fundamental Multidisciplinary Structure Technology with Application to an Innovative Gunner Restraint System for Improved Safety of Military Vehicles," Proceedings of the 16th Automotive Research Center Annual Conference, Ann Arbor, MI, May 2010.

${ }^{5}$ Bendsøe, M. P. and Kikuchi, N., "Generating Optimal Topologies in Structural Design Using a Homogenization Method," Computer Methods in Applied Mechanics and Engineering, Vol. 71, No. 2, 1988, pp. 197-224.

${ }^{6}$ Bendsøe, M. P., "Optimal Shape Design as a Material Distribution Problem," Structural Optimization, Vol. 1, No. 4, 1989, pp. 193-202.

${ }^{7}$ Bendsøe, M. P., Optimization of Structural Topology, Shape and Material, Springer, Berlin, 1995.

${ }^{8}$ Bendsøe, M. P. and Sigmund, O., Topology Optimization: Theory, Method and Applications, Springer, Berlin, 2003.

${ }^{9} \mathrm{Ma}$, Z. D., Kikuchi, N., and Cheng, H. C., "Topological Design for Vibrating Structures," Computer Methods in Applied Mechanics and Engineering, Vol. 121, No. 1, 1995, pp. 259-280.

${ }^{10} \mathrm{Ma}$, Z. D. and Kikuchi, N., "A New Method of the Sequential Approximate Optimization for Structural Optimization Problems," Engineering Optimization, Vol. 25, 1995, pp. 231-253.

${ }^{11}$ Sigmund, O., "A 99 Line Topology Optimization Code Written in Matlab," Structural Multidisciplinary Optimization, Vol. 21, No. 2, 2001, pp. 120-127.

${ }^{12}$ Zhou, M. and Rozvany, G. I. N., "The COC algorithm, part II: Topological, geometry and generalized shape optimization," Computer Methods in Applied Mechanics and Engineering, Vol. 89, No. 1, 1991, pp. 197-224.

${ }^{13}$ Brüls, O., Lemaire, E., and Duysinx, P., "Topology Optimization of Structural Components: A Multibody DynamicsOriented Approach," Proceeding of ECCOMAS Thematic Conference, Warsaw, Poland, 2009.

${ }^{14}$ Dong, G., Topology Optimization for Multi-Functional Components in Multibdy Dynamics Systems, Ph.D. Dissertation, The University of Michigan, Ann Arbor, 2012.

${ }^{15}$ Shabana, A. A., Dynamics of Mutibody Systems, Cambridge University Press, London, $2^{\text {nd }}$ ed., 1998.

${ }^{16}$ Dong, G., Ma, Z. D., Hulbert, G. M., Kikuchi, N., Arepally, S., Vunnam, M., and Lou, K., "An Efficient Optimal Design Methodology for Nonlinear Multibody Dynamics Systems with Application to Vehicle Occupant Restraint Systems," accepted by International Journal of Vehicle Design, 2011.

${ }^{17}$ Dong, G., Ma, Z. D., Hulbert, G. M., and Kikuchi, N., "Efficient Sensitivity Analysis for Multibody Dynamics Systems Using an Iterative Steps Method with Application in Topology Optimization," Proceedings of the ASME 2011 International Design Engineering Technical Conferences/Computers and Information in Engineering Conference, Washington, D.C., Aug. 2011.

${ }^{18}$ Dong, G., Ma, Z. D., Hulbert, G. M., and Kikuchi, N., "Time Inetegration Incoporated Sensitivity Analysis With Generalized- $\alpha$ Method For Multibody Dynamics Systems," Proceedings of the ASME 2011 International Mechanical Engineering Congress and Exposion, Denver, CO, Nov. 2011.

${ }^{19}$ Marler, R. T. and Arora, J. S., "Survey of Multi-Objective Optimization Methods for Engineering," Structural Multidisciplinary Optimization, Vol. 26, No. 6, 2004, pp. 369-395.

${ }^{20}$ Pareto, V., "Manuale di Economica Politica, Societa Editrice Libraria. Milan," translated into English in Manual of Political Economy 1927, 1906.

${ }^{21}$ Dasa, I., "An Improved Technique for Choosing Parameters for Pareto Surface Generation Using Normal-boundary Intersection," Proceedings of ISSMO/UBCAD/AIASA, the Third World Congress of Structural and Multidisciplinary Optimization, Buffalo, NY, Aug. 1999.

${ }^{22}$ Messac, A. A., Ismail-Yahaya, A., and Mattson, C. A., "The Normalized Normal Constraint Method for Generating the Pareto Frontier," Structural and Multidisciplinary Optimization, Vol. 25, No. 2, 2003, pp. 86-98.

${ }^{23}$ Holland, J. H., Adaptation in Natural and Artificial Systems, The University of Michigan Press, Ann Arbor, MI, 1975.

${ }^{24}$ Proos, K. A., Steven, G. P., Querin, O. M., and Xie, Y. M., "Multicriterion Evolutionary Structural Optimization Using the Weighting and the Global Criterion Methods," AIAA Journal, Vol. 39, No. 10, 2001, pp. 2006-2012.

${ }^{25}$ Koski, J. and Silvennoinen, R., "Norm Methods and Partial Weighting in Multicriterion Optimization of Structures," International Journal for Numerical Methods in Engineering, Vol. 24, No. 6, 2001, pp. 1101-1121.

${ }^{26}$ Rao, S. S. and Freiheit, T. I., "A Modified Game Theory Approach to Multiobjective Optimization," Journal of Mechanism Design, Vol. 113, No. 3, 1991, pp. 286-291.

${ }^{27}$ Hahn, H., Rigid Body Dynamics of Mechanisms - 1. Theoretical Basis, Springer, Berlin, 2002. 